\section{G员员傴 REPORT 89-6}

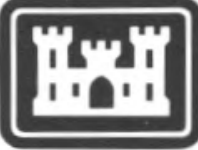

US Army Corps of Engineers

Cold Regions Research \&

Engineering Laboratory

Radiative transfer in falling snow

A two-stream approximation
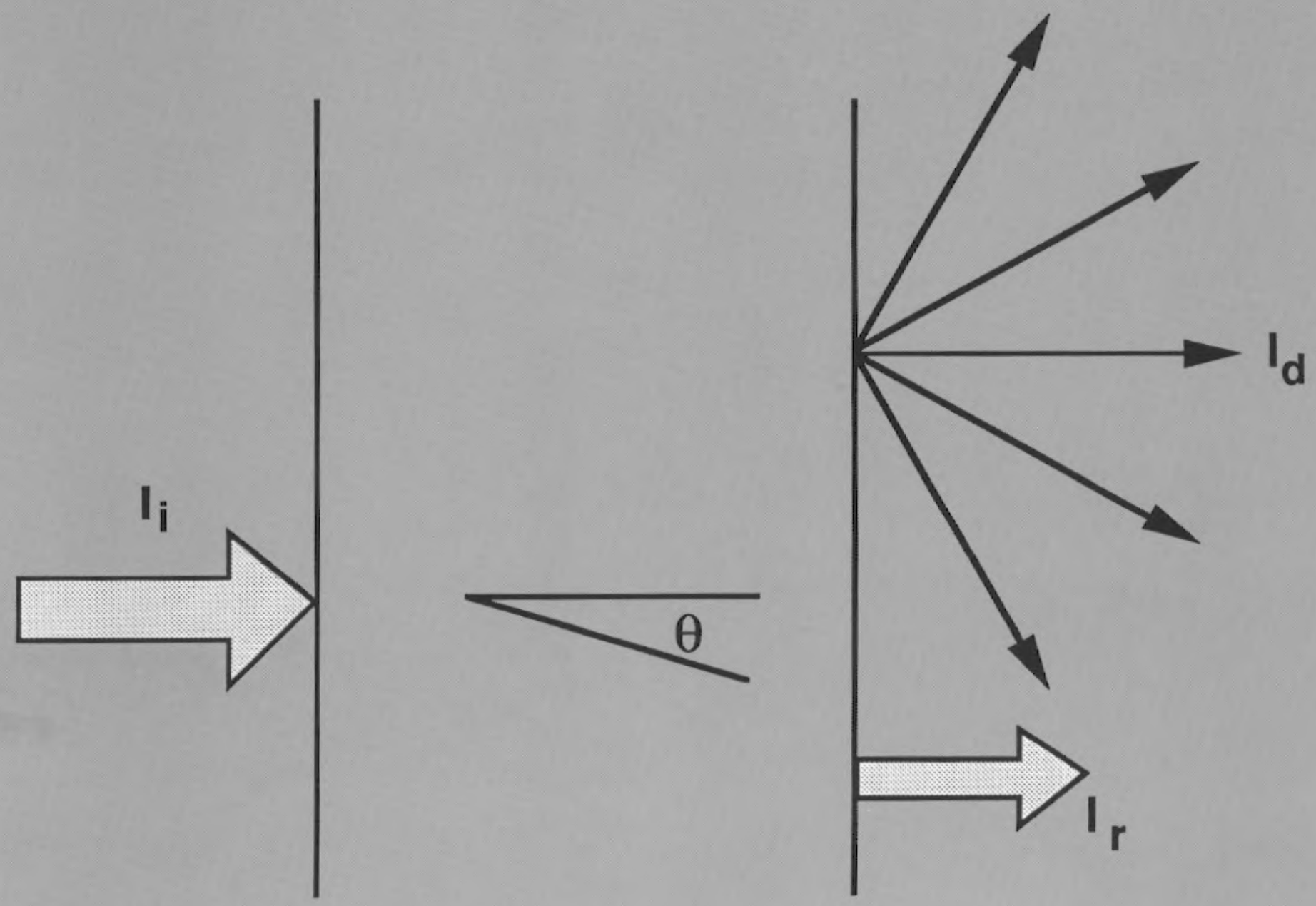
For conversion of SI metric units to U.S./British customary units of measurement consult ASTM Standard E380, Metric Practice Guide, published by the American Society for Testing and Materials, 1916 Race St., Philadelphia, Pa. 19103.

Cover: The intensity (1) of a beam of light incident on a snowfilled atmosphere is scattered $\left(1_{d}\right)$ and consequently reduced $\left(\hat{r}_{\mathrm{r}}\right)$. 


\section{CRREL Report 89-6}

April 1989

Radiative transfer in falling snow

A two-stream approximation

Gary Koh 
UNCLASSIFIED

SECURITY CLASSIFICATION OF THIS PAGE

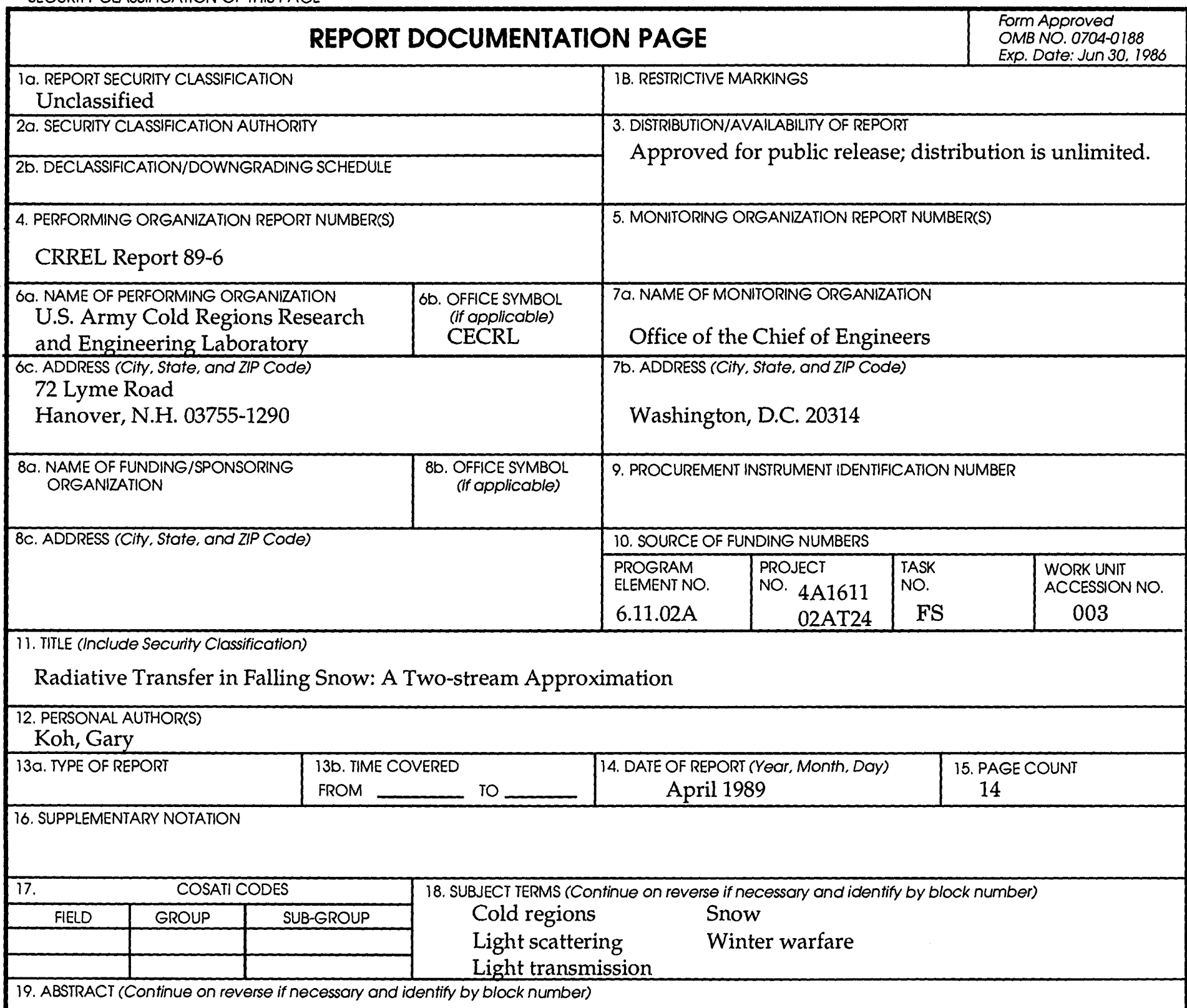

Light transmission measurements through falling snow have produced results unexplainable by single scattering arguments. A two-stream approximation to radiative transfer is used to derive an analytical expression that describes the effects of multiple scattering as a function of the snow optical depth and the snow asymmetry parameter. The approximate solution is simple and it may be as accurate as the exact solution for describing the transmission measurements within the limits of experimental uncertainties.

\begin{tabular}{|c|c|c|c|c|}
\hline $\begin{array}{l}\text { 20. DISTRIBUTION/AVAILABILITY OF ABSTRA } \\
\text { UNCLASSIFIED/UNLIMITED }\end{array}$ & $\begin{array}{l}\text { CT } \\
\text { SAME AS RPT. }\end{array}$ & $\square$ DTIC USERS & $\begin{array}{l}\text { 21. ABSTRACT SECURITY CLASSIFICATION } \\
\text { Unclassified }\end{array}$ & \\
\hline $\begin{array}{l}\text { 22a. NAME OF RESPONSIBLE INDIVIDUAL } \\
\text { Gary Koh }\end{array}$ & & & $\begin{array}{l}\text { 22b. TELEPHONE (Include Area Code) } \\
603-646-4100\end{array}$ & $\begin{array}{l}\text { 22c. OFFICE SYMBOL } \\
\text { CECRL-RG }\end{array}$ \\
\hline
\end{tabular}




\section{PREFACE}

This report was prepared by Gary Koh, Research Physical Scientist, Geophysical Sciences Branch, Research Division, U.S. Army Cold Regions Research and Engineering Laboratory. Funding for this research was provided by DA Project 4A161102 AT24, Research in Snow, Ice and Frozen Ground; Task Area FS, Fire Support; Work Unit 003, Microstructure of Hydrometeors.

The author thanks Dr. Donald K. Perovich and Roger H. Berger for their suggestions and technical reviews of this report.

The contents of this report are not to be used for advertising or promotional purposes. Citation of brand names does not constitute an official endorsement or approval of the use of such commercial products. 


\title{
Radiative Transfer in Falling Snow A Two-stream Approximation
}

\author{
GARY KOH
}

\section{INTRODUCTION}

The effects of multiple scattering must be considered to describe the transmission of visible light through an optically thick atmosphere containing haze, fog, clouds or solid aerosol particles. The rays or the photons that propagate through the atmosphere may undergo many scattering events before entering or escaping the field of view of a detector located downstream. Simple analytical expressions that are easy to interpret and that adequately represent the important features of the multiple scattering process can be obtained using various two-stream approximations to radiative transfer. Typically, the two-stream models parameterize the radiative properties of the atmosphere so that the intensities of the reflected and transmitted radiation can be calculated as a function of the optical depth, the single scattering albedo and the moments of the phase function.

The two-stream approximations are usually derived by beginning with Chandrasekhar's (1960) exact equation of radiative transfer and by making various approximations (Meador and Weaver 1980, King and Harshvardhan 1986). Bohren (1987) presents a derivation of a simple two-stream approximation in which the photons are constrained to be scattered strictly in the forward and backward directions. He avoids the use of Chandrasekhar's equation and gives a derivation similar to that of Schuster (1905), who initiated the two-stream radiation transfer theory long before the exact equation was developed. This report derives the two-stream approximation presented by Bohren (1987) by starting with the exact equation of radiative transfer to show the relationship between the approximate and the exact solutions. The simple two-stream theory is then used to describe the effects of multiple scattering on a parallel beam of light transmitted through falling snow. The comparison of the approximate results with the experimental results shows that the simple two-stream theory can be used to describe the transmission of visible light through falling snow.

\section{DESCRIPTION OF THE PROBLEM}

A parallel beam of light incident on a snow-filled atmosphere is scattered and absorbed so that the intensity of light reaching the detector located downstream decreases. To describe this decrease in intensity, it is convenient to distinguish between the incident intensity, $I_{\mathrm{i}}$, the reduced (unscattered and unabsorbed) intensity, $I_{\mathrm{r}}$, the diffuse (scattered) intensity, $I_{\mathrm{d}}$, and the total intensity, $I_{t}$. Figure 1 is a diagram illustrating the problem.

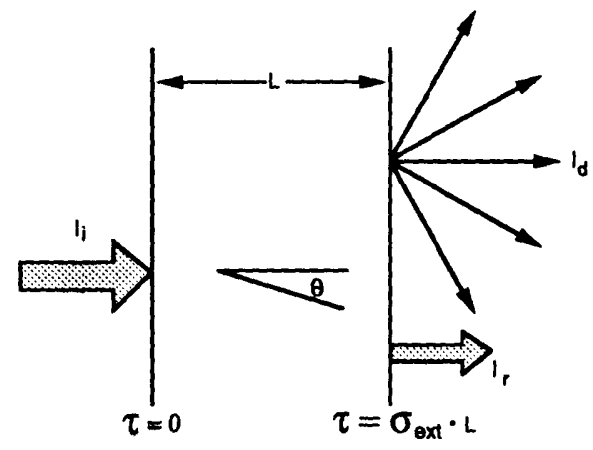

Figure 1. Relationship between the incident intensity $\left(a_{i}\right)$, the reduced intensity $\left(\mathrm{I}_{r}\right)$ and the diffuse intensity $\left(\mathrm{I}_{d}\right) \cdot \tau$ is the optical depth, $\mathrm{L}$ is the path length and $\mu=\cos \theta$. 
The reduced intensity is the portion of the incident light that travels the distance $L$ without undergoing scattering and absorption and satisfies the equation

$$
d I_{\mathrm{r}}=-I_{\mathrm{r}} \cdot \sigma_{\mathrm{ext}} \cdot d L
$$

where $\sigma_{\text {ext }}$ is the extinction coefficient. The extinction coefficient is defined as the sum of the absorption coefficient and the scattering coefficient. This equation integrates to yield the familiar Beer-Lambert law and the reduced intensity is expressed as

$$
I_{\mathrm{r}}=I_{\mathrm{i}} \exp (-\tau)
$$

where the optical depth, $\tau$, is defined as $\sigma_{\text {ext }} \cdot L$.

The diffuse intensity represents the portion of the incident light that is scattered one or more times and that travels in all directions according to the scattering characteristics of the snow particles. The equation that describes the transmission of diffuse intensity through a plane parallel atmosphere is the integro-differential equation of radiative transfer (Chandrasekhar 1960 , p. 22)

$$
\begin{aligned}
-\mu \frac{d}{d \tau} I_{\mathrm{d}}(\tau, \mu, \phi)= & I_{\mathrm{d}}(\tau, \mu, \phi)-\frac{1}{4 \pi} \int_{-1}^{1} \int_{0}^{2 \pi} I_{\mathrm{d}}\left(\tau, \mu^{\prime}, \phi^{\prime}\right) \varrho\left(\mu^{\prime}, \phi^{\prime} ; \mu, \phi\right) d \phi^{\prime} d \mu^{\prime} \\
& -I_{\mathrm{i}} \exp \left(-\tau / \mu_{0}\right) \varrho\left(-\mu_{0}, \phi_{0} ; \mu, \phi\right)
\end{aligned}
$$

where $\mu$ is the cosine of the angle $\theta$ measured from the axis normal to the parallel atmosphere, and $\phi$ is the corresponding azimuthal angle. The phase function $\varrho\left(\mu^{\prime}, \phi^{\prime} ; \mu, \phi\right)$ describes a single scattering event from the direction $\mu^{\prime}, \phi^{\prime}$ into the direction $\mu, \phi$. The incident beam comes from $-\mu_{0}$ direction..

The total intensity is the sum of the reduced intensity and the diffuse intensity and is expressed as

$$
I_{\mathrm{t}}=I_{\mathrm{r}}+I_{\mathrm{d}}
$$

From eq 2 and 3 it is seen that the reduced intensity diminishes exponentially while the behavior of the diffuse intensity is more complex. To avoid the extensive computations that are required to solve for the diffuse intensity, a simple two-stream approximation is used.

\section{TWO-STREAM APPROXIMATION}

The two-stream approximation used in this study is obtained by making several assumptions. It is initially assumed that the diffuse intensity is azimuthally independent and constant over the forward and the backward hemisphere (Schuster's approximation) so that

$$
I_{\mathrm{d}}(\tau, \mu, \phi)=\begin{array}{ll}
I_{\mathrm{d}}^{-}(\tau, \mu) ; & \mu>0 \\
I_{\mathrm{d}}^{-}(\tau, \mu) ; & \mu<0
\end{array}
$$

where $I_{\mathrm{d}}(\tau, \mu)$ and $I_{\mathrm{d}}^{-}(\tau, \mu)$ represent the intensities for the diffuse radiation in the forward and backward hemispheres respectively. The diffuse radiation is then constrained to travel in two directions only, exactly forward and exactly backward, so that the intensities can be expressed as $I_{\mathrm{d}}(\tau,+1)$ and $I_{\mathrm{d}}(\tau,-1)$ respectively. Applying these constraints to eq 3 results in the following two coupled linear differential equations 


$$
\begin{aligned}
& \frac{d}{d \tau} I_{\mathrm{d}}(\tau)=- I_{\mathrm{d}}(\tau)+F(-1 ;+1) I_{\mathrm{d}}(\tau)+F(+1 ;+1) I_{\mathrm{d}}(\tau) ; \mu=+1 \\
&+F(-1 ;+1) I_{\mathrm{i}} \exp (-\tau) \\
& \frac{d}{d \tau} I_{\mathrm{d}}^{-}(\tau)=I_{\mathrm{d}}^{-}(\tau)-F(+1 ;-1) I_{\mathrm{d}}^{-}(\tau)-F(-1 ;-1) I_{\mathrm{d}}(\tau) ; \mu=-1 \\
&-F(-1 ;-1) I_{\mathrm{i}} \exp (-\tau)
\end{aligned}
$$

where $I_{\mathrm{d}}(\tau)$ represents the light that comes from -1 direction and travels toward +1 direction and $I_{\mathrm{d}}(\tau)$ represents the light that comes from +1 direction and travels toward $-1 \mathrm{di}$ rection. $F\left(\mu^{\prime} ; \mu\right)$ is the fraction of light incident on a snow particle from the $\mu^{\prime}$ direction that is scattered in the $\mu$ direction. For example, $F(-1,-1)$ and $F(+1,+1)$ represent the fraction of the light that is scattered in the direction from which the light came (backscattering).

The values of $F\left(\mu^{\prime} ; \mu\right)$ approximate the integration in eq 3 for the forward and backward hemispheres so that

$$
\begin{aligned}
& F(-1 ;+1) \approx \frac{1}{4 \pi} \int_{-1}^{0} \int_{0}^{2 \pi} \varrho\left(\mu^{\prime}, \phi^{\prime} ; \mu, \phi\right) d \phi^{\prime} d \mu^{\prime} \\
& F(+1 ;+1) \approx \frac{1}{4 \pi} \int_{0}^{+1} \int_{0}^{2 \pi} \varrho\left(\mu^{\prime}, \phi^{\prime} ; \mu, \phi\right) d \phi^{\prime} d \mu^{\prime}
\end{aligned}
$$

$F(+1 ;-1)$ and $F(+1 ;+1)$ can be similarly represented.

The phase function of snow is not known, therefore the integration in eq $7 \mathrm{a}$ and $7 \mathrm{~b}$ must be carried out using some approximate phase function. The merits of performing such complex integrations using approximate values for the phase function are questionable. $F\left(\mu^{\prime} ; \mu\right)$ may be more conveniently approximated in terms of the asymmetry parameter, $g$, of the phase function and the single scattering albedo, $\omega_{0}$, so that (van de Hulst 1980)

$$
\begin{aligned}
& F(+1 ;-1)=F(-1 ;+1)=\omega_{0} \frac{(1+g)}{2} \\
& F(+1 ;+1)=F(-1 ;-1)=\omega_{0} \frac{(1-g)}{2} .
\end{aligned}
$$

The asymmetry parameter, $g$, is the mean cosine of the scattering angle and defines the degree of anisotropy of a phase function. The values for $g$ range from -1 to +1 .

For isotropic scattering and symmetrical scattering about $90^{\circ}$, the asymmetry parameter is 0 so that the values of $F\left(\mu^{\prime} ; \mu\right)$ in eq $8 \mathrm{a}$ and $8 \mathrm{~b}$ are one-half. In other words, an isotropic scatterer scatters one-half of the incident light in one direction and the other half in the opposite direction. For a highly anisotropic scatterer in the forward direction the value for $g$ approaches 1 . In this case eq $8 \mathrm{a}$ and $8 \mathrm{~b}$ approach 1 and 0 respectively. This means that most of the light is scattered in the direction of the incident beam (forward scattering). Similar reasoning can be applied to a scatterer whose asymmetry parameter approaches -1 .

By substitution of the results from eq $8 \mathrm{a}$ and $8 \mathrm{~b}$ into eq $6 \mathrm{a}$ and $6 \mathrm{~b}$, a two-stream model can be expressed by

$$
\frac{d}{d \tau} I_{\mathrm{d}}(\tau)=-I_{\mathrm{d}}(\tau)+\omega_{0} \frac{(1+g)}{2} I_{\mathrm{d}}(\tau)+\omega_{0} \frac{(1-g)}{2} I_{\mathrm{d}}^{-}(\tau)+\omega_{0} \frac{(1+g)}{2} I_{\mathrm{i}} \exp (-\tau)
$$




$$
\frac{d}{d \tau} I_{\mathrm{d}}^{-}(\tau)=I_{\mathrm{d}}^{-}(\tau)-\omega_{0} \frac{(1+g)}{2} I_{\mathrm{d}}^{-}(\tau)-\omega_{0} \frac{(1-g)}{2} I_{\bar{d}}(\tau)-\omega_{0} \frac{(1-g)}{2} I_{\mathrm{i}} \exp (-\tau)
$$

These coupled differential equations can be solved to determine the transmission of radiation using the boundary conditions $I_{\mathrm{d}}(0)=0$ at the start of the transmission path and $I_{\mathrm{d}}^{-}(\tau)$ $=0$ at the end of the transmission path.

A second set of two-stream approximations is derived from eq $9 \mathrm{a}$ and $9 \mathrm{~b}$, which are identical to those presented by Bohren (1987). This is done by suppressing the term containing the incident flux $I_{\mathrm{i}}$ so that the equations become

$$
\begin{aligned}
& \frac{d}{d \tau} I_{\mathrm{t}}^{-}(\tau)=-I_{\mathrm{t}}^{-}(\tau)+\omega_{0} \frac{(1+g)}{2} I_{\mathrm{t}}^{-}(\tau)+\omega_{0} \frac{(1-g)}{2} I_{\mathrm{t}}(\tau) \\
& \frac{d}{d \tau} I_{\mathrm{t}}(\tau)=I_{\mathrm{t}}^{-}(\tau)-\omega_{0} \frac{(1+g)}{2} I_{\mathrm{t}}(\tau)-\omega_{0} \frac{(1-g)}{2} I_{\mathrm{t}}^{-}(\tau)
\end{aligned}
$$

where the intensity $I_{\mathrm{t}}$ now refers to the total intensity $\left(I_{\mathrm{d}}+I_{\mathrm{r}}\right)$ and not just the diffuse intensity. Equations $10 \mathrm{a}$ and $10 \mathrm{~b}$ are solved (Appendix A) with the boundary conditions $I_{\mathfrak{t}}(0)=I_{i}$ and $I_{\mathrm{t}}^{-}(\tau)=0$ at the start and end of the transmission path, respectively, to yield the following expression for transmittance $T(\tau, g)$

$$
T(\tau, g)=\frac{I_{\mathrm{t}}^{-}(\tau)}{I_{\mathrm{i}}}=\frac{1}{1+\frac{(1-g)}{2} \tau} .
$$

This equation is used to describe the light transmission measurements that were made in falling snow.

\section{TRANSMISSION THROUGH SNOWFALL}

Visible light transmission data were obtained during snowfalls over a path distance of 650 $\mathrm{m}$. The source was a nearly parallel beam of light with a diameter of $0.6 \mathrm{~m}$ and a divergence of $20 \mathrm{mr}$. The receiver consisted of a detector located at the focal point of a $0.6-\mathrm{m}$-diameter lens with a field of view of $0.6 \mathrm{mr}$. During the transmission measurements, the snow mass concentration (snow mass per unit volume of air) was continuously measured at the midpoint of the transmission path.

Equation 11 shows that the transmission of visible light through falling snow can be determined if the optical path and the asymmetry parameter are known. The optical depth of snow-filled atmosphere is

$$
\tau=\sigma_{\mathrm{m}} \cdot M_{\mathrm{s}} \cdot L
$$

where $M_{\mathrm{s}}$ is the snow mass concentration, and the snow mass extinction coefficient, $\sigma_{\mathrm{m}}$, is defined as the extinction cross section per unit particle mass. The snow mass extinction coefficient is typically around $0.03 \mathrm{~m}^{2} / \mathrm{g}$ but it does vary depending on the snow crystal type.

The asymmetry parameter of snow was estimated to be 0.9 for this study. The value of 0.9 corresponds to the asymmetry parameter in the visible wavelength for an ice sphere with size similar to a snow particle. Although the spherical approximation for snow is an extreme idealization, it is used to obtain insights into the scattering process. Until the phase function 
of snow is known, the use of a spherical equivalent phase function may be the most appropriate approximation.

From eq 2, 4 and 11, the transmitted diffuse intensity can be expressed as

$$
I_{\vec{d}}(\tau, g)=\frac{I_{\mathrm{i}}}{1+\frac{(1-g)}{2} \tau}-I_{\mathrm{i}} \exp (-\tau) .
$$

The reduced intensity (eq 2 ) and the diffuse intensity components (eq 13) of the transmitted intensity are calculated as a function of snow mass concentration for a transmission path of $650 \mathrm{~m}$. The results that were calculated for a unit incident intensity are shown in Figure 2. It is seen that the diffuse intensity becomes dominant when the snow mass concentration exceeds $0.2 \mathrm{~g} / \mathrm{m}^{3}$, which is equivalent to an optical depth of 3.9 .

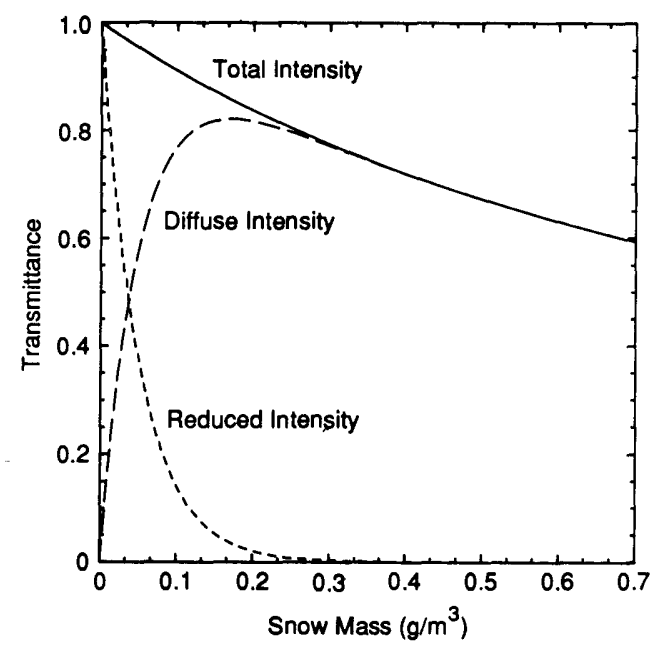

Figure 2. Relative contributions of the reduced intensity and the diffuse intensity to the total intensity as a function of snow mass concentration for a path distance of $650 \mathrm{~m}$.

Thus far it has been assumed that all the diffuse radiation transmitted in the forward direction contributes to the total intensity. However, because of the finite size of detectors used to measure the transmitted light and the spatial and angular spreading of the diffuse beam, only a fraction of the diffuse radiation is actually recorded. Therefore, in order to correctly interpret the experimental results, the measured diffuse radiation $I_{\mathrm{d}}^{\prime-}(\tau, g)$ is expressed as

$$
I_{\mathrm{d}}^{\prime}-(\tau, g)=C(\tau, g) \cdot I_{\vec{d}}(\tau, g)
$$

where the correction, $C(\tau, g)$, is the fraction of diffuse radiation that is actually measured. The measured transmittance, $T^{\prime}(\tau, g)$, is then expressed as

$$
T^{\prime}(\tau, g)=\exp (-\tau)+C(\tau, g) \cdot I_{\vec{d}}(\tau, g)
$$

The correction factor is dependent on the ratio of the effective detector area to the crosssectional area of the transmitted diffuse beam. In other words, for a given detector size, $C(\tau, g)$ decreases as the diffuse beam spreading increases. Therefore, information about diffuse beam spreading is required to determine the correction factor. The spreading of the diffuse beam is a complicated function of the optical depth and the snow phase function, therefore simplifying assumptions are made to determine the correction factor.

The diffuse beam diameter increases as the optical depth increases. If this increase is linear with respect to the optical depth, $C(\tau, g)$ will be inversely proportional to the square of the 
optical depth. The rate of increase is determined by the phase function; the beam spreading is less when the phase function is peaked in the forward direction. In other words, for a given optical depth, $C(\tau, g)$ will increase as the asymmetry parameter increases.

Another consideration for estimating $C(\tau, g)$ is the single scattering limit at low optical depths. At low optical depths, the scattered radiation that is measured is dominated by a sharp diffraction peak in the near-forward direction. Fraunhofer diffraction theory is commonly used to correct for the effects of single scattering on measured transmission (Seagraves 1983, Bohren and Koh 1985). These calculations indicate that for visible radiation, a significant portion of the light contained in the first diffraction lobe is measured by a typical transmission system (approximately 35 to $40 \%$ of all the scattered radiation is measured at low optical depths). From eq $8 \mathrm{~b}$ it is seen that a particle with an asymmetry parameter of 0.9 scatters $95 \%$ of the light in the forward hemisphere. Therefore, the limiting value of $C(\tau, g)$ at low optical depth (ratio of the scattered radiation that is measured to the radiation scattered in the forward direction) is expected to range from 0.37 to 0.42 . A correction factor of 0.4 is used for the low optical depth limit in this report.

By combination of the above-mentioned criteria required for the correction, the following expression is derived for the $C(\tau, g)$

$$
C(\tau, g)=\frac{0.40}{1+(1-g) \tau^{2}} .
$$

Figure 3 illustrates values for $C(\tau, g)$ for several asymmetry parameters. This figure shows that eq 16 satisfies the criteria discussed above. The values for $C(\tau, g)$ decrease as the optical depth increases, increase as the asymmetry parameter increases, and also approach the single scattering correction limit of 0.40 at the low optical depths.

The utility of any model is judged by its ability to predict experimental results. Figure 4 shows the comparison of the light transmission measured through falling snow with those calculated using the two-stream approximation with the correction factor applied. The figure shows that the experimental results can be described with reasonable acuracy without the use of an empricial adjustment. The transmission data presented in Figure 4 were selected so that the snow crystal type and size were similar through the range of the snow mass concentration.

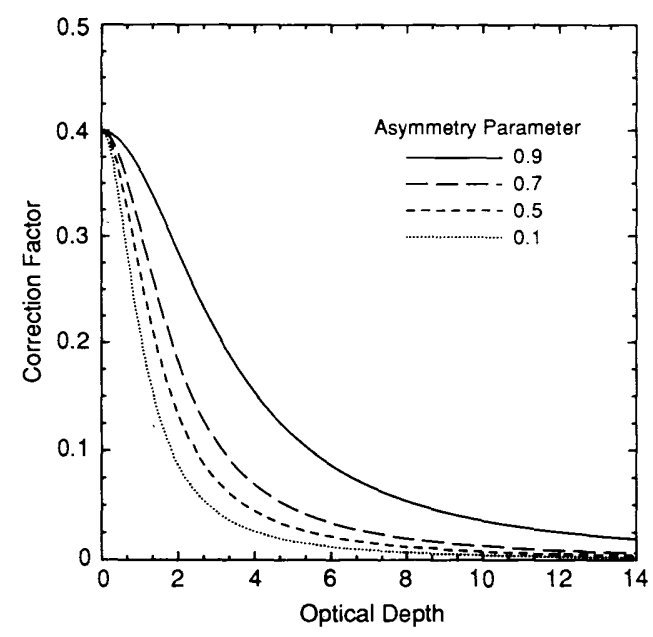

Figure 3. Correction for the fraction of diffuse intensity that is measured by a detector (eq 16). The asymmetry parameters $(\mathrm{g})$ are $0.9,0.7,0.5$ and 0.1 .

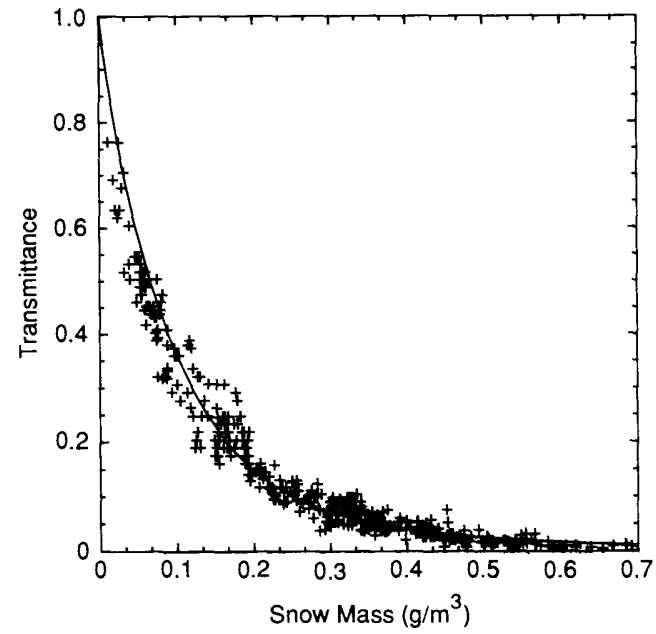

Figure 4. Comparison of the transmittance calculated from the two-stream approximation with the experimental results. The solid line represents the twostream results using eq 15 and 16. 
It is emphasized that the values for $C(\tau, g)$ were determined using qualitative arguments. The good agreement with the experimental results obtained using the qualitative arguments may be coincidental. More work is required to justify the analytical expression for $C(\tau, g)$. Nevertheless, it is important to point out that a correction factor that is qualitatively similar to the one presented is required to interpret light transmission measurements through an optically thick snowfall.

\section{CONCLUSIONS}

The parameters (the optical depth and the phase function) that are required for calculating the radiative properties of falling snow can only be approximated. Therefore, complex and time-consuming methods to solve the exact radiative transfer equations are not necessary when one is interested in interpreting experimental results. A simple two-stream approximation to radiative transfer where the light is constrained to travel in two directions only (forward and backward) is used to describe the transmission of visible light. A correction factor that accounts for the portion of the diffuse radiation that is actually measured by a detector is then applied. The comparison of the light transmission data obtained in snowfall with those calculated using the two-stream approximations is in good agreement over a wide range of optical depths.

\section{LITERATURE CITED}

Bohren, C.F. (1987) Multiple scattering of light and some of its observable consequences. American Journal of Physics, 55: 524-533.

Bohren, C.F. and G. Koh (1985) Forward-scattering corrected extinction by nonspherical particles. Applied Optics, 24: 1023-1029.

Chandrasekhar, S. (1960) Radiative Transfer. New York: Dover.

King, M.D. and Harshvardhan (1986) Comparative accuracy of selected multiple scattering approximations. Journal of Atmospheric Sciences, 43: 784-801.

Meador, W.E. and W.R. Weaver (1980) Two-stream approximations to radiative transfer in planetary atmosphere: A unified description of existing methods and a new improvement. Journal of Atmospheric Sciences, 37: 630-643.

Schuster, A. (1905) Radiation through foggy atmosphere. Astrophysics Journal, 21(1). (Reprinted in Selected Papers on the Transfer of Radiation [D.H. Menzel, Ed.]. New York: Dover, 1966.)

Seagraves, M.A. (1983) Visible and infrared transmission and precipitation rate in falling snow. Ph.D. Thesis, Colorado State University.

van de Hulst, H.C. (1980) Multiple Light Scattering. Volume 2. New York: Academic Press. 


\section{APPENDIX A: TWO-STREAM APPROXIMATION SOLUTION}

Adding and subtracting the following differential equations

$$
\begin{aligned}
& \frac{d}{d \tau} I_{\mathrm{t}}^{-}(\tau)=-I_{\mathrm{t}}^{-}(\tau)+\omega_{0} \frac{(1+g)}{2} I_{\mathrm{t}}^{-}(\tau)+\omega_{0} \frac{(1-g)}{2} I_{\mathrm{t}}^{-}(\tau) \\
& \frac{d}{d \tau} I_{\mathrm{t}}^{-}(\tau)=I_{\mathrm{t}}^{-}(\tau)-\omega_{0} \frac{(1+g)}{2} I_{\mathrm{t}}^{-}(\tau)-\omega_{0} \frac{(1-g)}{2} I_{\mathrm{t}}^{-}(\tau)
\end{aligned}
$$

result in the following:

$$
\begin{aligned}
& \frac{d}{d \tau}\left(I_{\mathrm{t}}^{-}+I_{\mathrm{t}}^{-}\right)=\left(1-\omega_{0} g\right)\left(I_{\mathrm{t}}^{-}-I_{\mathrm{t}}^{-}\right) \\
& \frac{d}{d \tau}\left(I_{\mathrm{t}}^{-}-I_{\mathrm{t}}^{-}\right)=\left(-1+\omega_{0}\right)\left(I_{\mathrm{t}}^{-}+I_{\mathrm{t}}^{-}\right) .
\end{aligned}
$$

For the case where absorption is neglible $\left(\omega_{0}=1\right)$, eq A2a and A2b become

$$
\begin{aligned}
& \frac{d}{d \tau}\left(I_{\mathrm{t}}^{-}+I_{\mathrm{t}}^{-}\right)=(1-g)\left(I_{\mathrm{t}}^{-}-I_{\mathrm{t}}^{-}\right) \\
& \frac{d}{d t}\left(I_{\mathrm{t}}^{-}-I_{\mathrm{t}}^{-}\right)=0 .
\end{aligned}
$$

The general solutions to eq $\mathrm{A} 3 \mathrm{a}$ and $\mathrm{A} 3 \mathrm{~b}$ are

$$
\begin{aligned}
& I_{\mathrm{t}}^{-}-I_{\mathrm{t}}^{-}=C_{1} \\
& I_{\mathrm{t}}^{-}+I_{\mathrm{t}}^{-}=C_{2}-(1-g) \cdot C_{1} \cdot \tau
\end{aligned}
$$

which can be reexpressed as

$$
\begin{aligned}
& I_{\mathrm{t}}^{-}=\frac{1}{2}\left(C_{1}+C_{2}\right)-\frac{(1-g)}{2} \cdot C_{1} \cdot \tau \\
& I_{\mathrm{t}}^{-}=\frac{1}{2}\left(C_{2}-C_{\mathrm{i}}\right)-\frac{(1-g)}{2} \cdot C_{1} \cdot \tau
\end{aligned}
$$

where constants $C_{1}$ and $C_{2}$ are determined by the boundary conditions.

Using the boundary conditions

$$
\begin{aligned}
& I_{\mathfrak{t}}-(\tau)=I_{\mathrm{i}} \text { for } \tau=0 \\
& I_{\mathfrak{t}}(\tau)=0 \text { for } \tau=\sigma_{\text {ext }} \cdot L
\end{aligned}
$$

the following is derived:

$$
\begin{aligned}
& C_{1}+C_{2}=2 I_{\mathrm{i}} \\
& C_{2}-C_{1}=(1-g) \cdot C_{1} \cdot \tau .
\end{aligned}
$$


These equations can be manipulated to show that

$$
C_{1}=\frac{I_{\mathrm{i}}}{1+\frac{(1-g)}{2} \cdot \tau} \text {. }
$$

The transmitted radiation, $T$, is then expressed as

$$
T=\frac{I_{\mathrm{t}}}{I_{\mathrm{i}}}=\frac{1}{1+\frac{(1-g)}{2} \cdot \tau}
$$


A facsimile catalog card in Library of Congress MARC format is reproduced below.

Koh, Gary

Radiative transfer in falling snow: A two-stream approximation / by Gary Koh. Hanover, N.H.: U.S. Army Cold Regions Research and Engineering Laboratory; Springfield, Va.: available from National Technical Information Service, 1989.

ii, 14 p., illus., $28 \mathrm{~cm}$. (CRREL Report 89-6.)

Bibliography: p. 7.

1. Cold regions. 2. Light scattering. 3. Light transmission. 4. Snow. 5. Winter warfare. I. United States Army. II. Corps of Engineers. III. Cold Regions Research and Engineering Laboratory. IV. Series: CRREL Report 89-6. 